\title{
ISOMETRIES ON $L_{p, 1}$
}

\author{
N. L. CAROTHERS AND B. TURETT
}

\begin{abstract}
The extreme points of the sphere of the Lorentz function space $L_{p, 1}[0,1]$ are computed. As an application, the linear isometries from $L_{p, 1}$ into itself are completely described.
\end{abstract}

1. Introduction. Since its introduction in 1950 by G. G. Lorentz, the Lorentz function space $L_{p, 1}=L_{p, 1}[0,1]$ has found frequent application to problems in interpolation theory and weighted-norm inequalities. In recent years the isomorphic structure of $L_{p, 1}$ (as a Banach space) has received increasing attention and is now reasonably well understood while very little has been written on the isometric structure of $L_{p, 1}$. In $\$ 2$, we develop several interesting isometric tools; in particular, we compute the extreme points of the closed unit ball of $L_{p, 1}$ (Theorem 1). As an application of these results, we give, in $\S 3$, a complete description of the linear isometries from $L_{p, 1}$ into itself (Theorem 2).

Recall that Lamperti's proof of Banach's classical theorem on the linear isometries $T: L_{p} \rightarrow L_{p}[18$, p. 333] proceeds in two major steps. In the first it is shown that $T$ must preserve disjointness (this via an observation concerning the $L_{p}$-norm). The second step is quite general: $T$ now induces a homomorphism of the measure algebra, and this homomorphism is necessarily induced by an automorphism, $\tau$, of the underlying measure space. It now follows easily that $T$ may be written: $T f=h \cdot(f \circ \tau)$, where $h=T 1$. Moreover, the converse is also true; that is, if $h$ is norm-one in $L_{p}$ and $\tau$ is an automorphism of $[0,1]$ with $\tau^{-1}[0,1]=$ support of $h$, then $T f=h \cdot(f \circ \tau)$ defines an isometry on $L_{p}$.

By modifying the first step of this argument, Bru and Heinich [2] are able to show that the positive (onto) isometries on a large class of Banach lattices (which includes $\left.L_{p, 1}\right)$ are likewise induced by automorphisms of the underlying measure space and so may also be written: $T f=h \cdot(f \circ \tau)$, where $h=T 1$. However, even in the case of $L_{p, 1}$, it is not clear whether the converse holds. Rather than deduce information for $L_{p, 1}$ from this lattice result, we opt for a somewhat more analytic approach to the question. We treat the isometries on $L_{p, 1}$ as an application of specific geometric and "distributional" tools intrinsic to $L_{p, 1}$. In particular, by using a stronger version of

Received by the editors July 2, 1985.

1980 Mathematics Subject Classification. Primary 46E30, 46B20.

Key words and phrases. Linear isometries, Lorentz function spaces, extreme points.

(C)1986 American Mathematical Society $0002-9947 / 86 \$ 1.00+\$ .25$ per page 
the first step outlined above (see Lemma 5, below), we shall see that there are actually fewer isometries on $L_{p, 1}$ than might be anticipated from the results in [2]. Specifically, not every norm-one $h$ in $L_{p, 1}$ can be written as $T 1$ for some isometry $T$.

We would like to thank Dale Alspach and William B. Johnson for several helpful conversations and especially for pointing out Lemma 3, below. We would also like to thank Lutz Weis for calling our attention to the work of Bru and Heinich [2].

Our notation is, for the most part, standard and follows that of Lindenstrauss and Tzafriri [15]. We write $\mu(A)$ for the Lebesgue measure of subset of $A$ of $\mathbf{R}$ and, given a measurable function $f:[0,1] \rightarrow \mathbf{R}$, we define

$$
\begin{aligned}
\operatorname{dist}(f ; t) & =d_{f}(t)=\mu(\{s:|f(s)|>t\}), \quad f^{*}(t)=\inf \left\{s: d_{f}(s) \leqslant t\right\}, \\
F(t) & =\int_{0}^{t} f^{*}(s) d s, \text { and } \operatorname{supp} f=\{s: f(s) \neq 0\} .
\end{aligned}
$$

Notice that $d_{f}$ is actually the (probability) distribution of $|f|$. Also we apologize in advance for all the usual abuses (and omissions) of the phrase "almost everywhere." For example, we shall sometimes write $f \geqslant 0$ when we mean $f \geqslant 0$ a.e., and $A \subset B$ instead of $\mu(B \backslash A)=0$, etc.

Now, for $1<p<\infty$, the Lorentz function space $L_{p, 1}=L_{p, 1}[0,1]$ is defined to be the collection of all (equivalence classes of) measurable functions $f:[0,1] \rightarrow \mathbf{R}$ for which $\|f\|_{p, 1}<\infty$ where

$$
\|f\|_{p, 1}=\int_{0}^{1} f^{*}(t) d\left(t^{1 / p}\right) .
$$

Simple change-of-variable and integration-by-parts arguments show that (1) can be written in a variety of guises:

$$
\begin{aligned}
\|f\|_{p, 1} & =\int_{0}^{\infty} d_{f}(t)^{1 / p} d t=\int_{0}^{1}\left(\frac{1}{p}\right) t^{1 / p-1} d F(t) \\
& =\int_{0}^{1} F(t) d\left(\left(\frac{-1}{p}\right) t^{1 / p-1}\right)+\|f\|_{1} .
\end{aligned}
$$

(Notice that if $f \in L_{p, 1}$, then $t^{1 / p-1} F(t) \rightarrow 0$ as $t \rightarrow 0^{+}$.) We shall also use the usual $L_{p}$-spaces with norm

$$
\|f\|_{p}=\left(\int_{0}^{1}|f(t)|^{p} d t\right)^{1 / p},
$$

and also the well-known duality: $\left(L_{p, 1}\right)^{*}=L_{p^{\prime}, \infty}, 1 / p+1 / p^{\prime}=1$, where

$$
\|f\|_{p^{\prime}, \infty}=\sup _{0<t<1} t^{-1 / p} \int_{0}^{t} f^{*}(s) d s .
$$

(See [16 or 13].)

It is also well known that $L_{p, 1}$ is a separable dual space [10] with an unconditional basis [15, p. 156]. Moreover, $L_{p, 1}$ is known to satisfy a lower $p$-estimate for disjoint elements [7, 1]; that is, if $f_{1}, \ldots, f_{n} \in L_{p, 1}$ are disjointly supported, then

$$
\left\|\sum_{i=1}^{n} f_{i}\right\|_{p, 1}^{p} \geqslant \sum_{i=1}^{n}\left\|f_{i}\right\|_{p, 1}^{p} .
$$

In particular, (4) implies that $\|f\|_{p} \leqslant\|f\|_{p, 1}$. (Also see [13 or 15, Proposition 2.6.9].) 
2. Extreme points. Our first two lemmas (which are essentially known) examine the triangle inequality in $L_{p, 1}$.

Lemma 1. If $f, g \in L_{p, 1}$ with $\|f+g\|_{p, 1}=\|f\|_{p, 1}+\|g\|_{p, 1}$, then $(f+g)^{*}=f^{*}$ $+g^{*}$.

Proof. First notice that since

$$
\|f\|_{p, 1}+\|g\|_{p, 1}=\|f+g\|_{p, 1} \leqslant\||f|+|g|\|_{p, 1} \leqslant\|f\|_{p, 1}+\|g\|_{p, 1},
$$

we must have $|f+g|=|f|+|g|$ a.e. Thus $f \cdot g \geqslant 0$ a.e. and $\|f+g\|_{1}=\|f\|_{1}+$ $\|g\|_{1}$. Now set

$$
F_{1}(x)=\int_{0}^{x}(f+g)^{*}(t) d t \quad \text { and } \quad F_{2}(x)=\int_{0}^{x}\left[f^{*}(t)+g^{*}(t)\right] d t
$$

for $0 \leqslant x \leqslant 1$. Then $F_{1} \leqslant F_{2}$ and we need to show that $F_{1}=F_{2}$. (See [15, p. 125].) But

$$
0=\|f\|_{p, 1}+\|g\|_{p, 1}-\|f+g\|_{p, 1}=\int_{0}^{1}\left[F_{2}(t)-F_{1}(t)\right] d\left(-\left(\frac{1}{p}\right) t^{1 / p-1}\right),
$$

and $-(1 / p) t^{1 / p-1}$ is increasing; thus $F_{2}-F_{1} \equiv 0$. Consequently, $(f+g)^{*}=f^{*}+$ $g^{*}$.

RemarK. Note that $(f+g)^{*}=f^{*}+g^{*}$ implies that $f \cdot g \geqslant 0$ and that

$$
\operatorname{supp}(f+g)^{*}=\operatorname{supp} f^{*} \cup \operatorname{supp} g^{*} \text {. }
$$

Thus

$$
\mu(\operatorname{supp} f \cup \operatorname{supp} g)=\max \{\mu(\operatorname{supp} f), \mu(\operatorname{supp} g)\} ;
$$

that is, we either have supp $f \subset \operatorname{supp} g$ or else $\operatorname{supp} g \subset \operatorname{supp} f$.

LEMMA 2. For $f \in L_{p, 1}$ and $a>0$, let $f^{a}=|f| \vee a-a$ and $f_{a}=|f| \wedge a$. Then $\|f\|_{p, 1}=\left\|f^{a}\right\|_{p, 1}+\left\|f_{a}\right\|_{p, 1}$. In particular, if $\|f\|_{p, 1}=1$ and $0<a<1$, then $\left\|f^{a}\right\|_{p, 1}$ $>0$ and $\left\|f_{a}\right\|_{p, 1}>0$, and hence $|f|$ is a convex combination of $f^{a} /\left\|f^{a}\right\|_{p, 1}$ and $f_{a} /\left\|f_{a}\right\|_{p, 1}$.

PROOF. A straightforward computation shows that $f^{*}=\left(f^{*}\right)^{a}+\left(f^{*}\right)_{a}=\left(f^{a}\right)^{*}$ $+\left(f_{a}\right)^{*}$ and so $\|f\|_{p, 1}=\left\|f^{a}\right\|_{p, 1}+\left\|f_{a}\right\|_{p, 1}$. Now, for $0<a<1$, it is easy to see that $0<a \cdot\left[d_{f}(a)\right]^{1 / p} \leqslant\left\|f_{a}\right\|_{p, 1} \leqslant a<1$, and hence also $\left\|f^{a}\right\|_{p, 1} \geqslant 1-a>0$.

We are now in a position to give a simple description of the extreme points of the closed unit ball of $L_{p, 1}$.

THEOREM 1. Let $1<p<\infty$ and let $f \in L_{p, 1}$ with $\|f\|_{p, 1}=1$. Then the following are equivalent:

(i) $\|f\|_{p}=1$.

(ii) $f$ is an extreme point of the closed unit ball of $L_{p, 1}$.

(iii) $|f|=\mu(E)^{-1 / p} \chi_{E}$ for some $E \subset[0,1]$.

(iv) $\left\||f|^{p-1}\right\|_{p^{\prime}, \infty}=1$, where $1 / p+1 / p^{\prime}=1$.

Proof. Suppose (i) holds. If $f=(g+h) / 2$ with $\|g\|_{p, 1}=\|h\|_{p, 1}=1$, then $\|g\|_{p}$ $=\|h\|_{p}=1$. The strict convexity of $L_{p}$ then implies $f=g=h$. 
Now suppose (ii) holds. Then, for any $0<a<1$, Lemma 2 implies that $f_{a}=\lambda|f|$ and $f^{a}=(1-\lambda)|f|$ where $\lambda=\left\|f_{a}\right\|_{p, 1}$. But this easily implies that $|f|=$ $\mu(E)^{-1 / p} \chi_{E}$ for some $E \subset[0,1]$.

That (iii) implies (iv) is obvious; so finally suppose (iv) holds. Then $\|f\|_{p}^{p-1}=$ $\left\||f|^{p-1}\right\|_{p^{\prime}} \geqslant\left\||f|^{p-1}\right\|_{p^{\prime}, \infty}=1$ and so $\|f\|_{p}=1$.

REMARK. The analogue of Theorem 1 for the Lorentz sequence space $l_{p, 1}$ is well known and is due to W. J. Davis (cf. e.g. [3]).

Since $L_{p, 1}$ is a separable dual space, its closed unit ball should have a wealth of strongly exposed points. (See [8 or 9].) As it happens, each extreme point of the closed unit ball of $L_{p, 1}$ is also strongly exposed. To see this, suppose $f \in L_{p, 1}$ is an extreme point of the closed unit ball and let $g=(\operatorname{sgn} f) \cdot|f|^{p-1}$. Then $\|f\|_{p}=\|g\|_{p^{\prime}}$ $=1=\langle f, g\rangle$ and $f$ (considered as an element of $L_{p}$ ) is strongly exposed by $g$ (considered as an element of $L_{p^{\prime}}$ ). Thus, if $\left\|f_{n}\right\|_{p, 1} \leqslant 1$ and $\left\langle f_{n}, g\right\rangle \rightarrow\langle f, g\rangle$, then $f_{n} \rightarrow f$ in $L_{p}$ and hence $\left\|f_{n}\right\|_{p} \rightarrow\|f\|_{p}=1$. But this implies that $\left\|f_{n}\right\|_{p, 1} \rightarrow\|f\|_{p, 1}=$ 1. That these conditions are sufficient to imply the convergence of the sequence $\left(f_{n}\right)$ to $f$ in $L_{p, 1}$ is given as

LEMMA 3. Let $\left(f_{n}\right)$ be a sequence in $L_{p, 1}$ such that $\left(f_{n}\right)$ converges to $f$ in $L_{p}$ and $\left(\left\|f_{n}\right\|_{p, 1}\right)$ converges to $\|f\|_{p, 1}$. Then $\left(f_{n}\right)$ converges to $f$ in $L_{p, 1}$.

Proof. First notice that it is enough to show that every subsequence of $\left(f_{n}\right)$ has a further subsequence converging to $f$ in $L_{p, 1}$. Consequently, we may assume that $\left(f_{n}\right)$ converges to $f$ a.e. Also, for convenience, we shall take $\|f\|_{p, 1}=1$. Now let $\varepsilon>0$ and choose $\delta>0$ so that $\left\|f \chi_{A^{c}}\right\|_{p, 1}<\varepsilon$ whenever $\mu(A)<\delta$. Next, use Egorov's theorem to choose $A$ so that $\mu(A)<\delta$ and so that $f_{n}$ converges uniformly to $f$ on $A^{C}$. Finally, choose $n$ sufficiently large so that the following hold:

(i) $\left\|\left(f-f_{n}\right) \chi_{A} c\right\|_{p, 1}<\varepsilon$,

(ii) $\left\|f_{n}\right\|_{p, 1}<1+\varepsilon$, and

(iii) $\left\|f_{n}\right\|_{p, 1}-\left\|f_{n} \chi_{A} c\right\|_{p, 1}<\varepsilon$.

Then

$$
\begin{aligned}
\left\|f-f_{n}\right\|_{p, 1} & \leqslant\left\|f \chi_{A}\right\|_{p, 1}+\left\|f_{n} \chi_{A}\right\|_{p, 1}+\left\|\left(f-f_{n}\right) \chi_{A^{c}}\right\|_{p, 1} \\
& \leqslant 2 \varepsilon+\left(\left\|f_{n}\right\|_{p, 1}^{p}-\left\|f_{n} \chi_{A^{c}}\right\|_{p, 1}^{p}\right)^{1 / p} \leqslant 2 \varepsilon+\left(p(1+\varepsilon)^{p-1} \varepsilon\right)^{1 / p}<2 p \varepsilon^{1 / p}
\end{aligned}
$$

Thus $\left(f_{n}\right)$ converges to $f$ in $L_{p, 1}$.

Before we can entertain any discussion of isometries on $L_{p, 1}$, we shall need some condition stated in terms of the norm in $L_{p, 1}$ which will guarantee that two functions are disjointly supported. The next two lemmas provide such conditions; the first is quite general (and really just a minor variation of Lemma 7.2 in [14]), while the second examines the case of equality in (4).

Lemma 4. Given $f, g \in L_{p, 1}$, let $f \oplus g$ denote the sum of disjoint copies of $f$ and $g$; that is, $d_{f \oplus g}=d_{f}+d_{g}$. (Of course, we may need to take $f \oplus g \in L_{p, 1}[0,2]$.) If $f \cdot g \geqslant 0$, then $\|f+g\|_{p, 1} \geqslant\|f \oplus g\|_{p, 1}$ and equality occurs only when $f \cdot g=0$. 
Proof. The first conclusion is a general fact in any rearrangement invariant space. Indeed, just as in the proof of Lemma 1, we need only to observe that if

$$
H_{1}(x)=\int_{0}^{x}(f \oplus g)^{*}(t) d t \quad \text { and } \quad H_{2}(x)=\int_{0}^{x}(f+g)^{*}(t) d t
$$

then $H_{1} \leqslant H_{2}$. But, since $f \cdot g \geqslant 0$,

$$
\begin{aligned}
H_{1}(x) & =\sup _{\mu E=X} \int_{E}|f \oplus g|(s) d s \leqslant \sup _{\mu E=X}\left\{\int_{E}|f(s)| d s+\int_{E}|g(s)| d s\right\} \\
& =\sup _{\mu E=X} \int_{E}|f+g|(s) d s=H_{2}(x) .
\end{aligned}
$$

Again, as in Lemma 1 , the case $\|f+g\|_{p, 1}=\|f \oplus g\|_{p, 1}$ would imply that $H_{1}=H_{2}$; that is $d_{f+g}=d_{f} \oplus g=d_{f}+d_{g}$. Then $f \cdot g \geqslant 0$ implies

$$
\mu(\operatorname{supp} f \cup \operatorname{supp} g)=d_{f+g}(0)=d_{f}(0)+d_{g}(0)=\mu(\operatorname{supp} f)+\mu(\operatorname{supp} g) \text {. }
$$

Thus $f \cdot g=0$.

LEMma 5. Let $f, g \in L_{p, 1}$ with $f \cdot g \geqslant 0$. If $\|f+g\|_{p, 1}^{p}=\|f\|_{p, 1}^{p}+\|g\|_{p, 1}^{p}$, then $f \cdot g=0$ and, moreover, $d_{f}$ and $d_{g}$ are proportional.

Proof. The first conclusion is immediate from (4) and Lemma 4. Indeed,

$$
\|f\|_{p, 1}^{p}+\|g\|_{p, 1}^{p}=\|f+g\|_{p, 1}^{p} \geqslant\|f \oplus g\|_{p, 1}^{p} \geqslant\|f\|_{p, 1}^{p}+\|g\|_{p, 1}^{p}
$$

and thus $f \cdot g=0$. But now

$$
\begin{aligned}
\left\{\int_{0}^{\infty}\left(d_{f}(t)+d_{g}(t)\right)^{1 / p} d t\right\}^{p} & =\|f+g\|_{p, 1}^{p}=\|f\|_{p, 1}^{p}+\|g\|_{p, 1}^{p} \\
& =\left\{\int_{0}^{\infty} d_{f}(t)^{1 / p} d t\right\}^{p}+\left\{\int_{0}^{\infty} d_{g}(t)^{1 / p} d t\right\}^{p} .
\end{aligned}
$$

That is, we have equality in the triangle inequality in $L_{1 / p}[0, \infty)$. Hence $d_{f}$ and $d_{g}$ are proportional. (In particular, $\|f\|_{p, 1}=\|g\|_{p, 1}$ would imply that $d_{f}=d_{g}$.)

REMARK. The observation made in [2] is that $L_{p, 1}$ is "order convex"; that is, if $f \cdot g \geqslant 0$ and if $\|f-g\|_{p, 1}=\|f+g\|_{p, 1}$, then $f \cdot g=0$. Notice that $f \cdot g \geqslant 0$ and $\|f+g\|_{p, 1}^{p}=\|f\|_{p, 1}^{p}+\|g\|_{p, 1}^{p}$ imply that $\|f+g\|_{p, 1}=\|f-g\|_{p, 1}$.

3. Isometries. Finally we are ready to describe the linear isometries from $L_{p, 1}$ into itself. What might not be expected here is that the only linear isometries are the obvious ones: changes of sign, rearrangements, and dilations. That is, if $T: L_{p, 1} \rightarrow$ $L_{p, 1}$ is an isometry and $\lambda=\mu(\operatorname{supp} T 1)$, then

$$
(T f)^{*}(t)=\lambda^{-1 / p} f^{*}(t / \lambda)
$$

for every $f \in L_{p, 1}$ and $0 \leqslant t \leqslant 1$. (We take $f^{*}(s)=0$ for $s>1$.)

Before we set a plan of attack for proving (5), let us first reduce to the case of positive isometries. In what follows, $T: L_{p, 1} \rightarrow L_{p, 1}$ is any linear isometry (not necessarily onto or positive) and, for each $n=1,2, \ldots$ and $i=1,2, \ldots, n, z_{n, i}$ will denote the characteristic function of the interval $[(i-1) / n, i / n)$. 
Lemma 6. Let $T: L_{p, 1} \rightarrow L_{p, 1}$ be a linear isometry. For every $f \in L_{p, 1}$, $\operatorname{supp} T f \subset \operatorname{supp} T 1$.

Proof. It suffices to show that (6) holds in the case $f=z_{n, i}$ for any $n>2^{p-1}$ and $i=1,2, \ldots, n$. To do this, it suffices to show that $T z_{n, i}$ and $T\left(1-z_{n, i}\right)$ are disjointly supported for all $n>2^{p-1}$ and all $i=1,2, \ldots, n$. Fix $n>2^{p-1}$ and $1 \leqslant i \leqslant n$, and set $f=T z_{n, i}, g=T\left(1-z_{n, i}\right), h=f+g=T 1, k=f-g=T\left(2 z_{n, i}-1\right)$. Then, since $T$ is an isometry, it is easy to see that

$$
\|f\|_{p, 1}=n^{-1 / p}, \quad\|g\|_{p, 1}=\left(1-n^{-1}\right)^{1 / p}, \quad\|h\|_{p, 1}=\|k\|_{p, 1}=1,
$$

and

$$
\begin{aligned}
& \|f+h\|_{p, 1}=\|f\|_{p, 1}+\|h\|_{p, 1}, \quad\|f+k\|_{p, 1}=\|f\|_{p, 1}+\|k\|_{p, 1}, \\
& \|g+h\|_{p, 1}=\|g\|_{p, 1}+\|h\|_{p, 1}, \quad\|g-k\|_{p, 1}=\|g\|_{p, 1}+\|k\|_{p, 1} .
\end{aligned}
$$

The equations in (8) follow from the fact that $\|1+x\|_{p, 1}=1+\|x\|_{p, 1}$ for any $x \in L_{p, 1}, x \geqslant 0$.

The equations in (8) combine with the remark following Lemma 1 to yield several consequences:

$$
f \cdot h \geqslant 0, \quad f \cdot k \geqslant 0, \quad g \cdot h \geqslant 0, \quad(-g) \cdot k \geqslant 0
$$

and

$$
\begin{aligned}
& \operatorname{supp} f \subset \operatorname{supp} h \quad \text { or } \operatorname{supp} h \subset \operatorname{supp} f, \\
& \operatorname{supp} f \subset \operatorname{supp} k \text { or } \operatorname{supp} k \subset \operatorname{supp} f \text {, } \\
& \operatorname{supp} g \subset \operatorname{supp} h \quad \text { or } \operatorname{supp} h \subset \operatorname{supp} g \text {, } \\
& \operatorname{supp} g \subset \operatorname{supp} k \quad \text { or } \operatorname{supp} k \subset \operatorname{supp} g \text {. }
\end{aligned}
$$

The inequalities in (9) imply that at any point for which $f \cdot g \neq 0$ we have $h \cdot k=0$ and, moreover, that $f \cdot g \geqslant 0$ on $\operatorname{supp} h$ and $f \cdot g \leqslant 0$ on $\operatorname{supp} k$. Consequently, if we set:

$$
\begin{aligned}
& A=\operatorname{supp} f \backslash \operatorname{supp} g, \\
& B=\operatorname{supp} g \backslash \operatorname{supp} f, \\
& C=\operatorname{supp} h \backslash(A \cup B)=\{\operatorname{sgn} f=\operatorname{sgn} g\}, \\
& D=\operatorname{supp} k \backslash(A \cup B)=\{\operatorname{sgn} f=-\operatorname{sgn} g\},
\end{aligned}
$$

then $A, B, C, D$ are pairwise disjoint and

$$
\begin{array}{lr}
f=f \chi_{A} & +\frac{1}{2} h \chi_{C}+\frac{1}{2} k \chi_{D}, \\
g= & g \chi_{B}+\frac{1}{2} h \chi_{C}-\frac{1}{2} k \chi_{D} .
\end{array}
$$

But now (10) implies several conditions on $A, B, C$, and $D$. In fact, it is not hard to see that either $A=B=\varnothing$ or else $C=D=\varnothing$ (that is, either $h \cdot k=0$ or $f \cdot g=0$ ). Indeed, suppose for instance that $A \neq \varnothing$ and $C \neq \varnothing$. Then $\operatorname{supp} g=B$ $\cup C \cup D$ and $\operatorname{supp} k=A \cup B \cup D$ cannot satisfy either of the containments $\operatorname{supp} g \subset \operatorname{supp} k$ or supp $k \subset \operatorname{supp} g$. Thus we need only point out that $A=B=\varnothing$ (i.e., $h \cdot k=0$ ) is impossible. But $\|h+k\|_{p, 1}=2\|f\|_{p, 1}=2 n^{-1 / p}$ while from (4), $h \cdot k=0$ would imply $\|h+k\|_{p, 1} \geqslant 2^{1 / p}$; our choice of $n>2^{p-1}$ makes this impossible. Thus $f \cdot g=0$ as desired. 
LEMma 7. Let $T: L_{p, 1} \rightarrow L_{p, 1}$ be a linear isometry. The map $S: L_{p, 1} \rightarrow L_{p, 1}$ defined by $S f=(\operatorname{sgn} T 1) \cdot T f$ is a positive isometry. In particular, iff $\cdot g \geqslant 0$, then $T f \cdot T g \geqslant 0$.

Proof. As mentioned above, for any $f \geqslant 0$, we have $\|T 1+T f\|_{p, 1}=\|T 1\|_{p, 1}+$ $\|T f\|_{p, 1}$ and so, from Lemma $1, T 1 \cdot T f \geqslant 0$. Since Lemma 6 states that supp $T f \subset$ $\operatorname{supp} T 1$, we can conclude that $(\operatorname{sgn} T 1) \cdot T f=|T f|$.

Our next goal will be to provide a more tractable (i.e., linear) replacement for (5). To this end, it may be helpful to think of $T$ as an isometry from $L_{p, 1}$ into $L_{p, 1}\left([0,1]^{2}\right)$. The reasons for this are essentially cosmetic: if we define $f \otimes g$ by $(f \otimes g)(s, t)=f(s) g(t)$, then it is easy to see that the map $f \rightarrow f \otimes g$ defines an isometry satisfying (5) whenever $|g|=\mu(E)^{-1 / p} \chi_{E}$. Indeed, the distribution of $f \otimes \chi_{E}$ is $\mu(E) \cdot d_{f}$ and so, in this case,

$$
(f \otimes g)^{*}(t)=\mu(E)^{-1 / p} f^{*}(t / \mu(E)) .
$$

Now it is also known that

$$
\|f\|_{p, 1} \cdot\|g\|_{p} \leqslant\|f \otimes g\|_{p, 1} \leqslant\|f\|_{p, 1} \cdot\|g\|_{p, 1}
$$

for any $f, g \in L_{p, 1}[4,5$ and 17, Theorem 7.4] and so our program is easy to outline. We shall first show that $T f$ must have same distribution as $f \otimes g$ where $g=T 1$. We shall then show that $g=T 1$ forces equality in (14). This implies that $\|g\|_{p}=\|g\|_{p, 1}$ $=1$ or equivalently, by Theorem 1 , that $g=\mu(E)^{-1 / p} \chi_{E}$ for some measurable set E.

LEMMA 8. Let $T: L_{p, 1} \rightarrow L_{p, 1}$ be a linear isometry and let $g=T 1$. Then $T f$ has the same distribution as $f \otimes g$ for every $f$ in $L_{p, 1}$.

Proof. Since $T$ is linear and continuous and since step functions of the form $\sum_{i=1}^{n} a_{i} z_{n, i}$ are dense in $L_{p, 1}$, it is enough to show that, for each $n$, the functions $T z_{n, i}, i=1,2, \ldots, n$, are disjointly supported and have the same distribution. Fix $n$ and $1 \leqslant i \neq j \leqslant n$. Since $T$ is a linear isometry, we have $\left\|T z_{n, i}\right\|_{p, 1}=\left\|T z_{n j}\right\|_{p, 1}$ and $\left\|T z_{n, i}+T z_{n, j}\right\|_{p, 1}^{p}=\left\|T z_{n, i}\right\|_{p, 1}^{p}+\left\|T z_{n, j}\right\|_{p, 1}^{p}$. But now Lemma 5 and Lemma 7 give us that $T z_{n, i}$ and $T z_{n, j}$ are disjointly supported and have the same distribution. Necessarily $T z_{n, i}$ has the same distribution as $z_{n, i} \otimes g$ and linearity implies that $T\left(\sum_{i=1}^{n} a_{i} z_{n, i}\right)$ has the same distribution as $\left(\sum_{i=1}^{n} a_{i} z_{n, i}\right) \otimes g$.

Next we consider the case of (near) equality on the left-hand side of inequality (14). The following lemma is suggested by the proof of Lemma 8.8 of [14]. (Also see [15, Theorem 2.7.2 and 6].)

LEMMA 9. Given a positive integer $k$ and $\alpha>1$, define $f_{k, \alpha} \in L_{p, 1}$ by $f_{k, \alpha}(t)=k^{\alpha / p}$ $\wedge t^{-1 / p}$. Then, for any $h=\sum_{i=1}^{k} a_{i} z_{k, i}$,

$$
\left\|f_{k, \alpha} \otimes h\right\|_{p, 1} \leqslant\left(1+\alpha^{-1}\right) \cdot\|h\|_{p} \cdot\left\|f_{k, \alpha}\right\|_{p, 1} .
$$

Proof. We shall show that if $\|h\|_{p} \leqslant 1$, then $\left(f_{k, \alpha} \otimes h\right)^{*} \leqslant f_{k, \alpha+1}$; (15) then follows from a simple calculation:

$$
\begin{aligned}
\left\|f_{k, \alpha+1}\right\|_{p, 1} & =1+\frac{\alpha+1}{p} \log k \leqslant\left(1+\alpha^{-1}\right) \cdot\left(1+\frac{\alpha}{p} \log k\right) \\
& =\left(1+\alpha^{-1}\right) \cdot\left\|f_{k, \alpha}\right\|_{p, 1} .
\end{aligned}
$$


Assume that $h=\sum_{i=1}^{k} a_{i} z_{k, i}$ satisfies $h \geqslant 0$ and $\|h\|_{p}^{p}=k^{-1} \sum_{i=1}^{k} a_{i}^{p} \leqslant 1$; we wish to estimate $\left(f_{k, \alpha} \otimes h\right)^{*}$. But $f_{k, \alpha} \otimes h=\sum_{i=1}^{k} a_{i}\left(f_{k, \alpha} \otimes z_{k, i}\right)$ and the functions $f_{k, \alpha}$ $\otimes z_{k, i}$ are disjointly supported and have distribution $k^{-1} \operatorname{dist}\left(f_{k, \alpha} ; t\right)$. Thus

$$
\operatorname{dist}\left(f_{k, \alpha} \otimes h ; t\right)=k^{-1} \sum_{i=1}^{k} \operatorname{dist}\left(f_{k, \alpha} ; \frac{t}{a_{i}}\right) .
$$

Now $\operatorname{dist}\left(f_{k, \alpha} ; t\right)=t^{-p} \wedge \chi_{\left[0, k^{\alpha / p}\right)}(t)$, and it is easy to check that, for $0<a \leqslant k^{1 / p}$, we have

$$
\operatorname{dist}\left(f_{k, \alpha} ; t / a\right)=a^{p} t^{-p} \wedge \chi_{\left[0, k^{\alpha / p}\right)}(t / a) \leqslant \chi_{[0,1)}(t)+a^{p} t^{-p} \chi_{\left[1, k^{(\alpha+1) / p}\right)}(t) .
$$

Consequently,

$$
\begin{aligned}
\operatorname{dist}\left(f_{k, \alpha} \otimes h ; t\right) & =k^{-1} \sum_{i=1}^{k} \operatorname{dist}\left(f_{k, \alpha} ; \frac{t}{a_{i}}\right) \\
& \leqslant \chi_{[0,1)}(t)+\left(k^{-1} \sum_{i=1}^{k} a_{i}^{p}\right) \cdot t^{-p} \cdot \chi_{\left[1, k^{(\alpha+1) / p}\right)}(t) \\
& \leqslant t^{-p} \wedge \chi_{\left[0, k^{(\alpha+1) / p}\right)}(t)=\operatorname{dist}\left(f_{k, \alpha+1} ; t\right)
\end{aligned}
$$

Thus, $\left(f_{k, \alpha} \otimes h\right)^{*} \leqslant f_{k, \alpha+1}$.

We are finally ready to combine all of the preceding observations to give a simple proof of our main result.

TheOREM 2. Let $T: L_{p, 1} \rightarrow L_{p, 1}$ be a linear isometry and let $\lambda=\mu(\operatorname{supp} T 1)$. Then T satisfies

$$
(T f)^{*}(t)=\lambda^{-1 / p} f^{*}(t / \lambda)
$$

for every $f$ in $L_{p, 1}$ and $0 \leqslant t \leqslant 1$.

Proof. Let $g=T 1$. Lemma 8 shows that $(T f)^{*}=(f \otimes g)^{*}$ for every $f \in L_{p, 1}$. Thus, by (13) and Theorem 1 , we need only show that $\|g\|_{p}=1$.

Let $0<\varepsilon<1$ and let $h=\sum_{i=1}^{k} a_{i} z_{k, i}$ be a step function such that $\|g-h\|_{p, 1} \leqslant$ $\varepsilon\|g\|_{p}$. Next let $f=f_{k, 1 / \varepsilon}$ be the function given in Lemma 9 (for $\alpha=1 / \varepsilon$ ). Then

$$
\begin{aligned}
\|f\|_{p, 1} & =\|T f\|_{p, 1}=\|f \otimes g\|_{p, 1} \leqslant\|f \otimes h\|_{p, 1}+\|f \otimes(g-h)\|_{p, 1} \\
& \leqslant(1+\varepsilon)\|h\|_{p} \cdot\|f\|_{p, 1}+\|(g-h)\|_{p, 1} \cdot\|f\|_{p, 1} \\
& \leqslant\left[(1+\varepsilon)^{2}+\varepsilon\right] \cdot\|g\|_{p} \cdot\|f\|_{p, 1}
\end{aligned}
$$

(where the second inequality follows from (14) and (15)). Letting $\varepsilon$ tend to 0 yields $\|g\|_{p}=1$ as promised.

REMARK. Notice that every linear isometry on $L_{p, 1}$ turns out to be an isometry on $L_{p}$ and, in fact, a multiple of an isometry on every $L_{r}$. Thus a linear isometry $T$ on $L_{p, 1}$ maps extreme points to extreme points and is onto exactly when $|T 1|=1$. 


\section{REFERENCES}

1. Z. Altshuler, The modulus of convexity of Lorentz and Orlicz sequence spaces, Notes in Banach Spaces (H. E. Lacey, ed.), Univ. of Texas Press, 1980.

2. B. Bru and H. Heinich, Isometries positives et propriétés ergodiques de quelques espaces de Banach, Ann. Inst. H. Poincaré Sect. B 17 (1981), 377-405.

3. J. R. Calder and J. B. Hill, A collection of sequence spaces, Trans. Amer. Math. Soc. 152 (1970), 107-118.

4. N. L. Carothers, Rearrangement invariant subspaces of Lorentz function spaces, Israel J. Math. 40 (1981), 217-228.

5. __ Rearrangement invariant subspaces of Lorentz function spaces. II, preprint.

6. N. L. Carothers and P. H. Flinn, Embedding $l_{p}^{n^{\alpha}}$ into $l_{p, q}^{n}$, Proc. Amer. Math. Soc. 88 (1983), $523-526$.

7. J. Creekmore, Type and cotype in Lorentz $L_{p, q}$ spaces, Indag. Math. 43 (1981), 145-152.

8. M. M. Day, Normed linear spaces, Ergeb. Math. Grenzgeb., Bd. 21, Springer-Verlag, 1973.

9. J. Diestel and J. J. Uhl, Jr., Vector measures, Math. Surveys, no. 15, Amer. Math. Soc., Providence, R. I., 1977.

10. I. Halperin, Function spaces, Canad. J. Math. 5 (1953), 273-288.

11. G. Hardy, J. E. Littlewood and G. Polya, Inequalities, Cambridge Univ. Press, 1952.

12. E. Hewitt and K. Stromberg, Real and abstract analysis, Graduate Texts in Math. 25, Springer-Verlag, 1965.

13. R. A. Hunt, On $L(p, q)$ spaces, Enseign. Math. 12 (1966), 249-274.

14. W. B. Johnson, B. Maurey, G. Schechtman and L. Tzafriri, Symmetric structures in Banach spaces, Mem. Amer. Math. Soc. No. 217, 1979.

15. J. Lindenstrauss and L. Tzafriri, Classical Banach spaces. II, Ergeb. Math. Grenzgeb., Bd. 97, Springer-Verlag, 1979.

16. G. G. Lorentz, Some new functional spaces, Ann. of Math. 51 (1950), 37-50.

17. R. O'Neil, Integral transforms and tensor products on Orlicz spaces and $L(p, q)$ spaces, J. Analyse Math. 21 (1968), 1-276.

18. H. L. Royden, Real analysis, Macmillan, 1968.

Department of Mathematics, Oklahoma State University, Stillwater, Oklahoma 74078

Department of Mathematical Sciences, OAKland University, Rochester, Michigan 48063 (Current address of B. Turett)

Current address (N. L. Carothers): Department of Mathematics, Texas A \& M University, College Station, Texas 77843 